One abnormal high blood pressure may not be indicative of hypertension in young people with diabetes. If blod pressure recodings are multiple and persistently raised, ambulatory blood pressure monitoring may need to be considered.

\section{G436(P) AUDIT AND QUALITY IMPROVEMENT OF THE NATIONAL HEALTH SERVICE ENGLAND (NHSE) SPECIALISED SERVICE FOR CHILDREN WITH ALSTROM SYNDROME}

${ }^{1} \mathrm{M}$ Charalambides, ${ }^{2} \mathrm{M}$ Kershaw, ${ }^{2} \mathrm{~J}$ Pemberton, ${ }^{3} \mathrm{~K}$ Brock, ${ }^{1,2} \mathrm{~T}$ Barrett. ${ }^{1}$ Medical School, College of Medical and Dental Sciences, University of Birmingham, Birmingham, UK; ${ }^{2}$ Endocrinology and Diabetes, Birmingham Women's and Children's Hospital, Birmingham, UKi ${ }^{3}$ Cancer Research UK Clinical Trials Unit, University of Birmingham, Birmingham, UK

\subsection{6/archdischild-2020-rcpch.377}

Background Alstrom syndrome is a rare $(1: 1,000,000)$ multisystem condition associated with infancy-onset obesity and insulin-resistant diabetes during puberty. It is unknown whether deterioration of renal function begins in childhood and whether this relates to glycaemic control.

Primary Aims To audit children attending the national service $(n=24)$ against the standard of annual BMI, renal, liver function, and glucose control and compare to the 2018 audit.

To design BMI-SDS graphs to allow clearer indication of BMI progress.

Methods Case notes and laboratory data were reviewed for 2015-2019. eGFR was calculated using the Counahan-Barratt method.

Results Screening of blood pressure, U\&Es and urine albumin creatinine measurements (target level:80\%) has improved from $10 / 24(42 \%)$, in the first audit cycle, to $15 / 22(68 \%)$.

$11 / 20(55 \%)$ had a reduction in eGFR (median decline 25 $\mathrm{ml} / \mathrm{min} / \mathrm{yr}$, range:9-58 $\mathrm{ml} / \mathrm{min} / \mathrm{yr}$ ) (declining eGFR group) compared to $9 / 20(45 \%)$ (stable eGFR group). Comparing the declining eGFR to the stable eGFR group, median age was 14.5 yrs (4-17.9 yrs) vs 10.3 yrs (6.5-16.8 yrs); 6/11 (55\%) had Hba1c $\geq 6.5 \%$ (median $7.3 \%$ (5.7-7.7)) vs 9/20 (45\%); 7/11 (64\%) were overweight ( $\geq 2$ BMI-SDS) vs $6 / 9$ $(67 \%) ; 4 / 11(36 \%)$ were obese (>3 BMI-SDS) vs $1 / 9$ (11\%); and $8 / 11(73 \%)$ were of South Asian origin vs $3 / 9$ (33\%).

The average BMI-SDS remained at >2.67SDS (>99.6th centile) in the Asian cohort of Alstrom patients over the 4 years, but decreased from $>2.67$ SDS to $1.5 \mathrm{SDS}$ in the White European cohort.

Conclusions Higher BMI-SDS, South Asian origin, older age and poorer glycaemic control were associated with a reduction in eGFR over time. The co-occurrence of these features at puberty may relate to a 'metabolic syndrome' phenotype associated with high levels of insulin resistance known to occur in mid puberty. The results for the South Asian cohort suggest the tendency for the phenotype to remain obese into adulthood.

Recommendations Prospective studies should investigate the drivers to loss of glycaemic control and reduction in eGFR around puberty and the reasons for the ethnic differences in BMI-SDS. Improved monitoring in childhood and earlier pharmacologic and lifestyle interventions may be required to prevent eGFR deterioration and achieve better glycaemic control. Bespoke BMI-SDS charts may empower children and families for weight management. Culturally specific dietetic advice may be helpful.

\section{G437 VALIDITY OF THE WASHINGTON GROUP MODULE ON CHILD FUNCTIONING IN 2-YEAR-OLD CHILDREN; DISABILITY OUTCOME OF THE SHINE TRIAL}

${ }^{1} \mathrm{~T}$ Dunne, ${ }^{1,2}{ }^{2}$ Chandna, ${ }^{2} \mathrm{~F}$ Majo, ${ }^{2} \mathrm{~B}$ Chasekwa, ${ }^{2} \mathrm{R}$ Ntozini, ${ }^{2,3,4} \mathrm{~A}$ Prendergast, ${ }^{2,4} \mathrm{~J}$ Humphrey, ${ }^{1} \mathrm{M}$ Gladstone, ${ }^{2} \mathrm{~N}$ Tavengwa, ${ }^{2} \mathrm{~B}$ Mutasa. ${ }^{1}$ Faculty of Health and Life Sciences, University of Liverpool, Liverpool, UK; ${ }^{2}$ Institute for Maternal and Child Health Research, Zvitambo, Harare, Zimbabwe; ${ }^{3}$ Blizard Institute, Queen Mary University of London, London, UK; ${ }^{4}$ Bloomberg School of Public Health, Johns Hopkins, Baltimore, Maryland, USA

\subsection{6/archdischild-2020-rcpch.378}

Introduction The WHO estimate that 1 billion people live with disability, with $80 \%$ living in developing countries. For children, this is associated with increased mortality, risk of becoming a victim of violence and decreased school attendance. Through identifying these children early, actions can be taken to improve their quality of life.

Global childhood disability data collection to date has used various tools and methodologies, affecting comparability. In 2016, the Washington Group and UNICEF developed the child functioning module (WGCFM) to identify functional difficulty (FD) in children aged $2-17$, for use in census and surveys. Field testing has highlighted a need for further validation. This study aims to identify prevalence of disability according to the WGCFM in over 1500 2-year-old children from the Sanitation Hygiene Infant Nutrition Study (SHINE) trial in 2 districts of Zimbabwe, and to assess concurrent validity with the Malawi Developmental Assessment Tool (MDAT).

Methods All consenting pregnant females aged 15-49 were followed up throughout pregnancy and infancy. At age 2, their child was assessed for disability by one of 11 local research nurses using both the WGCFM and MDAT (a culturally appropriate tool for developmental assessment in rural Africa - adapted, translated and back translated for use in Zimbabwe). Quality control and reliability tests were performed on all measures between research nurses. Other measures included anthropometry (e.g. height, weight), maternal HIV status and behavioural measures.

Results 1606 (49.9\% female) participants from SHINE were eligible for disability follow-up. FD was identified in $4.17 \%$, severe FD in $0.68 \%$. MDAT and WGCFM scores showed a significant negative correlation, $\mathrm{P}<0.001$. Those with FD had a significantly lower MDAT score, $\mathrm{P}=0.012$; this became more significant when comparing those with severe FD to no FD, $\mathrm{P}=0.002$

Conclusions Following removal of the 'controlling behaviour' domain, prevalence of FD by WGCFM in rural Zimbabwe is comparable to that in field testing in Mexico, Samoa and Serbia. These results demonstrate that the WGCFM has concurrent validity for use within a population of 2-year-old children in Zimbabwe. 